\title{
The categorization of natural scenes: Brain attention networks revealed by dense sensor ERPs
}

\author{
Maurizio Codispoti, ${ }^{\mathrm{a}, *}$ Vera Ferrari, ${ }^{\mathrm{b}}$ Markus Junghöfer, ${ }^{\mathrm{c}}$ and Harald T. Schupp ${ }^{\mathrm{d}}$ \\ ${ }^{a}$ Department of Psychology, University of Bologna, Italy \\ ${ }^{\mathrm{b}}$ Department of Human and General Physiology, University of Bologna, Italy \\ ${ }^{\mathrm{c}}$ Institute for Biomagnetism and Biosignalanalysis, Münster University Hospital, Germany \\ ${ }^{\mathrm{d}}$ Department of Psychology, University of Konstanz, Germany
}

Received 14 September 2005; revised 28 February 2006; accepted 5 April 2006

\begin{abstract}
The present study examined cortical indicators of selective attention underlying categorization based on target features in natural scenes. The primary focus was to determine the neural sources associated with the processing of target stimuli containing animals compared to nontarget control stimuli. Neural source estimation techniques [current source density (CSD) and L2-minimum norm estimate (L2-MNE)] were used to determine the sources of the potential fields measured from 58 sensor sites. Assuring an excellent signal-to-noise ratio, the categorization task consisted of 2400 trials. Replicating previous findings, target and non-target ERP activity diverged sharply around $150 \mathrm{~ms}$ after stimulus onset and the early differential ERP activity appeared as positive deflection over fronto-central sensor sites and as negative deflection over temporo-occipital regions. Both source estimation techniques (CSD and L2-MNE) suggested primary sources of the early differential ERP activity in posterior, visual-associative brain regions and, although less pronounced, revealed the contribution of additional anterior sources. These findings suggest that selective attention to category-relevant features reflects the interactions between prefrontal and inferior temporal cortex during visual processing of natural scenes.
\end{abstract}

\section{Introduction}

Selective attention processes towards task-relevant stimuli facilitate the efficient organization of goal-directed behavior. Experimentally, target detection tasks serve to explore the neural systems involved in selective attention. By definition, detection tasks implicate the encoding and comparison of relevant stimulus and target-defining features. In separate lines of event-related brain

\footnotetext{
* Corresponding author. Fax: +39 51243086.

E-mail address: maurizio.codispoti@unibo.it (M. Codispoti).
}

potential studies, individuals performed target detection tasks, which were defined either according to selected stimulus features (e.g., size, color, or shape) or, more recently, higher-order stimulus categories.

Several ERP components have been associated with the processing of target compared to non-target stimuli in detection tasks defining the target stimuli according to specific stimulus features such as size, color, shape and orientation (Baas et al., 2002). Consistently, a posterior negativity was found to be associated with target processing, which was observed about 150-300 ms poststimulus and variously referred to as selection negativity, N2, N2pc or N2b (Harter and Aine, 1984; Näätänen, 1992; Potts and Tucker, 2001; Smid et al., 1999). The latency and topography of this posterior negativity is dependent upon the target defining features and therefore considered to reflect activity in neural stimulus representations representing target defining visual features. In the same latency range, there is also a positive deflection (P2) over frontal regions, variously referred to as $\mathrm{P} 2 \mathrm{a}$, P2f or frontal selection positivity (Kenemans et al., 1993; Potts and Tucker, 2001; Smid et al., 1999). N2 and P2 differ in their sensitivity to experimental manipulations, and therefore the $\mathrm{P} 2$ is not viewed to simply reflect polarity reversal of the N2. Specifically, whereas N2 and P2 amplitudes are enhanced to target stimuli, the P2 amplitude is insensitive to manipulations of stimulus frequency (Potts et al., 1996). It has therefore been suggested that in visual object target detection, the P2 and N2 components reflect the interaction between prefrontal and inferior temporal cortex (Potts, 2004) with prefrontal regions generating top-down signals reflected in differential perceptual processing of target and non-target items (cf. Kastner et al., 2000; Wood and Grafman, 2003).

Using complex natural scenes, more recent studies explored detection tasks in which the target stimuli were defined by higherorder category information (Delorme et al., 2000; Fabre-Thorpe et al., 2001; Thorpe et al., 1996; VanRullen and Thorpe, 2001a). For instance, Thorpe et al. (1996) asked subjects to categorize briefly presented natural scenes according to whether they contained an 
animal or not. Despite the complexity of the pictures and categorization task, reaction time was less than $400 \mathrm{~ms}$. Furthermore, ERP measurements revealed that target animal compared to control stimuli started to differentiate around $150 \mathrm{~ms}$ after stimulus presentation, and the differential ERP activity (target minus nontarget) was most pronounced around $200 \mathrm{~ms}$ poststimulus. Emphasizing the speed of target detection in this category detection task, this ERP component has been dubbed "differential ERP activity" calculated as the difference between target and non-target stimuli. The differential ERP activity reflecting target processing is observed as negative potential over temporo-occipital and positive deflection over frontal sensor regions (Rousselet et al., 2002, 2004). Further studies observed a similar differential ERP activity when the target category was defined by artificial objects (means of transport) rather than biologically relevant (natural) target categories (VanRullen and Thorpe, 2001a).

Due to methodological limitations, that is, limited numbers of sensors and reference-dependent characterization of field potential (Tucker et al., 1994), the neural sources of the early differential ERP activity in natural scene categorization tasks are currently not fully explored. Initially, the early differential ERP activity was considered to reflect anterior neural generator sites implicated in the inhibition of inappropriate behavioral responses during NoGo trials (Thorpe et al., 1996). However, modifying the behavioral task rendered this specific hypothesis unlikely (Antal et al., 2000; De Cesarei et al., 2006; Johnson and Olshausen, 2003). A more recent hypothesis assumes that the early differential ERP activity reflects posterior generators in the visual ventral processing stream. In accordance with this reasoning, a recent study by Delorme et al. (2004) reported bilateral generators of the differential ERP activity in temporo-occipital regions. However, it is not currently determined whether the anterior positivity/posterior negativity reflects neural generators located in visual-associative cortices or activity in prefrontal and inferior temporal cortex as was observed for target detection based on specific stimulus features.

In a recent study, Johnson and Olshausen (2005) observed that the differential ERP activity was obtained for photographic objects as well as words, and was reduced by degradation of the target stimulus or a change in the semantic level of categorization. Based on these findings, Johnson and Olshausen (2005) suggested that the early differential ERP activity is a component of the classical P3 wave. According to this hypothesis, the early differential ERP activity is related to the increased stimulus value of target stimuli in working memory rather than selective target processing in modality-specific perceptual. Although interesting, spatial undersampling of the scalp potentials and reference-dependent characterization of the field potential (right mastoid) limited the assessment of topography of the early differential ERP component. However, to explore this hypothesis, the present study also measured the P3 wave, appearing with positive polarity over centro-parietal sensor in a time window from 300 to $700 \mathrm{~ms}$. Although considered the most studied ERP component in detection tasks using specific stimulus characteristics, the P3 component was not the focus of previous research using natural scene categorization tasks. The few studies exploring the P3 wave are inconclusive because, using the Go/NoGo paradigm, target and non-target stimuli were unbalanced in terms of motor activation. In order to compare neural generators of the early differential ERP activity and P3 wave, the present study eliminated asymmetries in motor demands by asking participants to respond to target and non-target stimuli.
The present study examined cortical indicators of selective attention underlying categorization based on target features in natural scenes. The primary focus was to determine the neural sources associated with the processing of target stimuli containing animals compared to non-target control stimuli. Based on Delorme et al. (2004), we expected that the differential ERP activity to reflect neural sources in temporo-occipital regions. In addition, we explored whether posterior activations fully account for the differential ERP activity or, as was the case in detection tasks based on specific stimulus features, we obtain evidence for anterior (prefrontal) sources contributing to the differential ERP activity. Cortical neural generators were estimated by calculating the current source density (CSD) and L2-minimum norm estimate (L2-MNE; Hamalainen and Ilmoniemi, 1994; Hauk et al., 2002). As this technique requires high spatial sampling of the scalp potentials (cf. Tucker et al., 1994), the ERP was collected in this study from 58 sensor sites. Furthermore, to avoid spurious findings due to low signal-to-noise ratio, the categorization task consisted of 2400 trials assuring an excellent signal-to-noise-ratio (Picton et al., 2000). Because both neural source estimation techniques rely on different assumptions (Hauk, 2004; Junghöfer et al., 1997), converging evidence of the CSD and L2-MNE solutions were considered particularly informative, preventing spurious findings related to limitations inherent in each source estimation technique. A secondary aim of the present study was to distinguish the early differential ERP from a scalp-recorded neural activity in the classical P3 time region. In particular, we expected the classical P3 to be distinct form the early differential ERP activity in terms of scalp topography and neural generators estimated by modeling techniques.

\section{Materials and methods}

\section{Participants}

Seventeen right-handed students from the University of Padova (nine males with ages ranging from 21 to 28 years) participated in this study. All participants had normal or corrected to normal eyesight. All subjects gave inform consent after the nature of the experiment was explained and the study was approved by the University of Padova ethics committee.

\section{Stimulus materials and procedure}

Following several previous studies (Fize et al., 2000; Thorpe et al., 1996; VanRullen and Thorpe, 2001b), stimuli were natural color photographs taken from a large commercially available CDROM library allowing access to several thousand stimuli. The images in the animal target and non-animal non-target category were selected to be as varied as possible. Thus, the animal target category included 1200 complex pictures containing mammals as well as birds, fishes, insects and reptiles. The non-animal nontarget category included 1200 complex scenes such as outdoor or indoor images, natural landscapes, street scenes with buildings and roads, pictures of food, plants, houses and man-made objects or tools. Each picture was shown for $24 \mathrm{~ms}$. The interstimulus interval (ISI) varied between 1.5 and $2 \mathrm{~s}$.

The subject's task was to press one of the two buttons with the index or middle finger of the right hand, indicating whether the presented image contained an animal or not (two alternative 
forced-choice task). Target and non-target items were presented with a probability of $50 / 50 \%$. Sequences of at most three pictures of each category were allowed. The subjects had no a priori information on the size, position or number of target animals in the single target photographs.

Each subject participated in two sessions. In each session, the subjects viewed a total of 1200 pictures. Across both sessions, each of the 2400 pictures was shown only once avoiding image-specific learning effects. Within a session, pictures were shown in four blocks of 300 pictures separated by a 5 -min resting period.

\section{Apparatus and data analysis}

\section{Behavioral responses}

E-Prime software (Schneider et al., 2002) was used to present the pictorial stimuli and record the behavioral responses. For target and non-target items, the proportion of correct versus incorrect responses as well as response times was calculated and submitted to a repeated measures ANOVA, including the factor category (target vs. non-target). For reaction times, response latencies longer than twice the individual mean were rejected as outliers.

\section{Event-related potentials}

The electroencephalogram (EEG) was recorded from 58 sites according to the international 10-20 system using a Neuroscan amplifier and data acquisition software. Scalp impedance for each sensor was kept below $5 \mathrm{k} \Omega$. All channels were recorded with a $\mathrm{Cz}$ reference and converted off line to an average reference. The EEG as well as vertical and horizontal eye movements (EOG) was recorded continuously with a sampling rate of $1000 \mathrm{~Hz}$ and on-line band-pass filtered from 0.01 to $100 \mathrm{~Hz}$. Continuous EEG and EOG data were low-pass filtered at $30 \mathrm{~Hz}$ using digital filtering before stimulus synchronized epochs were extracted from $100 \mathrm{~ms}$ before until $1000 \mathrm{~ms}$ after picture onset. All individual trials with EEG or EOG activity above $\pm 50 \mu \mathrm{V}$ were excluded from further analysis. On average, 960 out of the 1200 trials for each condition were retained in the analysis assuring a good signal-to-noise ratio. To correct for eye blinks, we applied an artifact correction to single trial EEG epochs (Semlitsch et al., 1986). Finally, separate average waveforms were calculated for each picture category (target and non-target) for each sensor and participant.

Two complementary analyses were conducted to analyze the differential ERP activity to target and non-target items. First, following Thorpe et al. (1996) and VanRullen and Thorpe (2001b), Student's $t$ tests (target vs. non-target) were calculated for each time point after picture onset separately for each individual sensor in order to identify the temporal and spatial characteristics of the selective processing of target pictures. These waveform analyses were conducted using a significance criterion of $P<0.01$. In order to avoid false positives, significant effects were only considered meaningful, when the effects were observed for at least fifteen continuous data points $(15 \mathrm{~ms})$. Second, in order to provide a formal test including the topography of the effects, we calculated repeated measures ANOVA. Accordingly, sensors were grouped into 6 clusters (see Fig. 1): left frontal (FP1, AF3, F1, F3, F5, F7), right frontal (FP2, AF4, F2, F4, F6, F8), left centro-parietal (C1, $\mathrm{C} 3, \mathrm{CP} 1, \mathrm{CP} 3, \mathrm{P} 1, \mathrm{P} 3)$, right centro-parietal (C2, C4, CP2, CP4, P2, P4), left occipito-temporal (TP7, P5, P7, PO3, PO5, PO7, O1) and right occipito-temporal (TP8, P6, P8, PO4, PO6, PO8, O2). Early and late differential ERP activities were scored as mean activity in the time intervals from 150 to $200 \mathrm{~ms}$ and 350 to 400 ms, respectively. Repeated measures analyses of variance (MANOVA) were calculated including the factors stimulus category (target vs. non-target), site (frontal vs. centro-parietal vs. occipitotemporal) and laterality (left vs. right).

\section{Source estimation}

Current source density (CSD). The calculation of the CSD as well as the fundamentally equivalent methods "Laplacian" or "intracranial potential mapping (IPA)" are mathematically unique transformations and serve to compensate for the strong spatial lowpass filtering effect of the head as volume conductor-predominantly the "blurring" effect of the skull. These "deblurring" methods do not demand any a priori constraints or assumptions, are not affected by the ambiguity of the "inverse problem" and thus served for uncovering cortical generator sources. Although mathematically unique, the goodness of all "deblurring" methods heavily depend on a sufficient spatial sampling as well as an adequate signal to noise ratio (Junghöfer et al., 1997). With ERP measurements at 58 sensor sites and approximately 1000 trials for each condition, the present study met these requirements. Here, we used the CSD approach as recommended by Junghöfer et al. (1997), which is based on a physiological volume conductor model and well suited for usage of dense array EEG. In CSD solutions, a focal generator source is indicated by a sink/source pattern of inward/outward flow of current, which, however, reveals a more complicated distribution of multiple inward and outward currents in the case of activation of adjacent generator sources. Thus, although this method has limitations in attributions of sink/source patterns to specific neural structures, the technique is sufficiently sensitive to the question to what extent the early differential ERP activity reflects anterior and posterior generator sources.

Minimum norm analyses. A second source estimation technique was used to provide further independent evidence regarding the generator source of the early differential ERP activity. The L2minimum norm estimate enables enhanced resolution of scalprecorded field potentials without a priori assumptions regarding the location and/or number of current sources. Although the L2-MNE does not provide precise anatomical localizations, resolution should provide meaningful information regarding the issue to what extent the early differential ERP activity reflects primarily posterior or anterior as well as posterior brain generators.

The minimum norm, as a linear estimation technique, is based on the assumption that the measured scalp potential distribution $(U)$ at each point in time can be described as the product of a leadfield matrix (L), specifying each electrode sensitivity to each of the distributed sources of the model head, and the generator activation $(G) \Rightarrow U=\mathbf{L} G$. In order to estimate the source distribution $G=\mathbf{L}^{-}$ $U$, the inverse of the leadfield matrix $\mathbf{L}$ has to be multiplied with the measured scalp potential distribution. However, this matrix inversion is only defined if the number of columns (given by the number of sensors in $U$ ) and rows (given by the number of sources in $G$ ) of $\mathbf{L}$ would be identical and $\mathbf{L}$ would have maximal rank. With distributed source models, the number of sources strongly exceeds the number of sensors, and thus $\mathbf{L}^{-}$has to be replaced by the pseudoinverse leadfield matrix $\mathbf{L}^{+}$, leading to $G=\mathbf{L}^{+} U$. The pseudoinverse multiplication is characterized by minimizing the power (L2) of the estimated current density of the sources. Here we used a spherical shell with evenly distributed 3 (radial, azimuthal and polar direction) $\times 197$ dipoles as source model. A shell radius of $6 \mathrm{~cm}$ has been 


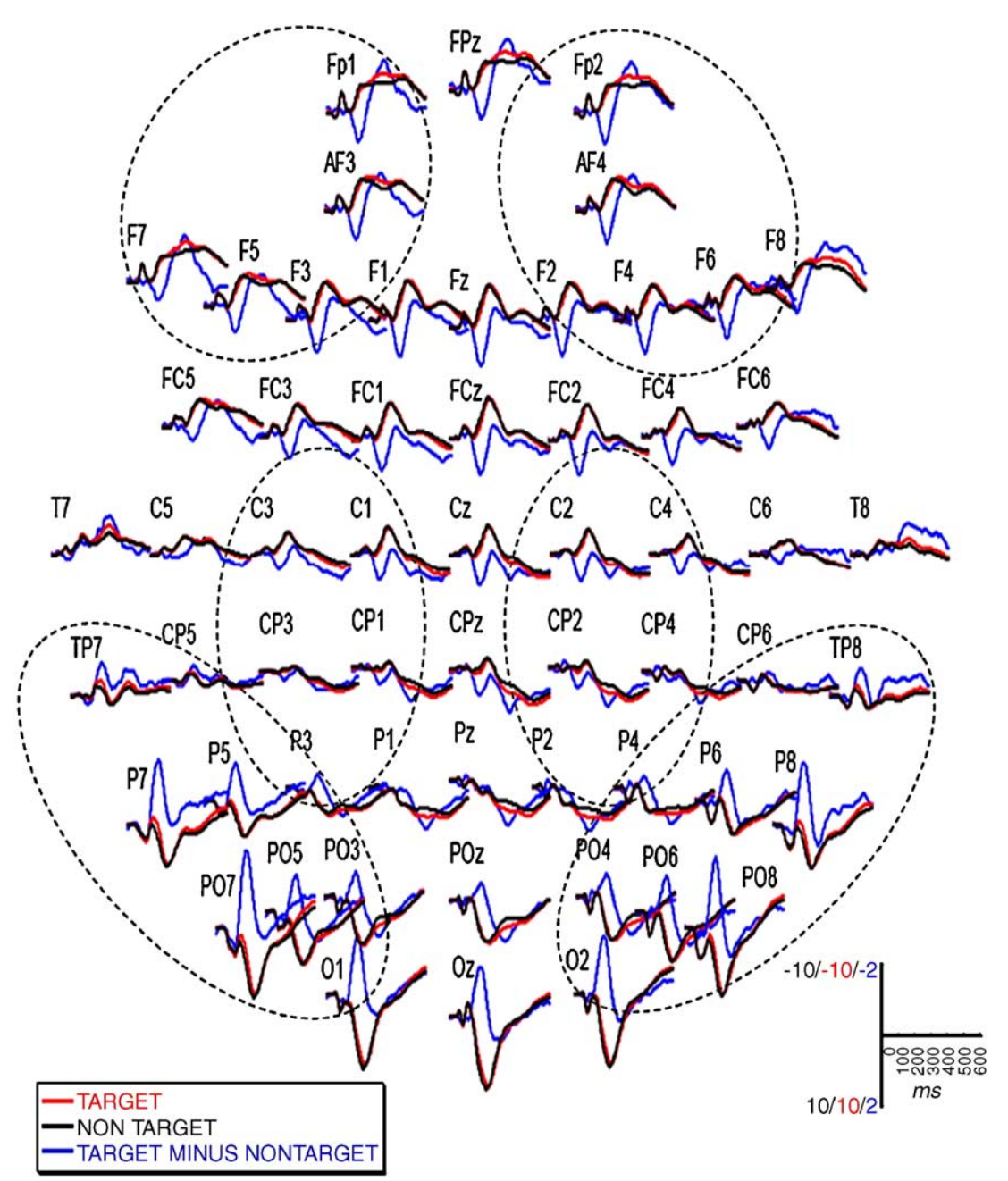

Fig. 1. Grand-averaged ERP waveforms while viewing target and non-target pictures indicated by red and black colored lines, respectively. In addition, the difference waveforms (target-non-target images) are represented by blue lines. Please note that the difference waveform is shown on a different scale to adjust for magnitude differences. The dashed circles indicate the sensors contributing to the average frontal, centro-parietal and occipito-temporal averages displayed in Fig. 2.

chosen as a tradeoff between depth sensitivity and spatial resolution (Hauk et al., 2002). As a consequence of underestimated source covariance, minimum norm solutions may be prone to spurious inverse solutions. To provide the best trade-off between the stability of the inverse solution and spatial resolution, we applied a "Tihkonov regularization" to the pseudoinverse matrix, specifically selected to the present data. To empirically determine the optimal "Tihkonov regularization", we plotted the root mean square of difference between the original and the estimated data (inverse/ forward calculated) across a range of regularization parameters (Lcurve fitting; Hansen, 1992). Sharp bends in the individual L-curved graphs suggested optimal regularization and resulted in an average regularization parameter of 0.01 in the present study.

\section{Results}

\section{Behavioral data}

As expected, response times to target pictures $(M=545 \mathrm{~ms} ; \mathrm{SD}=$ 53.7) were faster compared to non-target items $(M=575 \mathrm{~ms}$; $\mathrm{SD}=$ 59.5), category, $F(1,16)=34.9, P<0.001$. Furthermore, as in previous studies, responses were quite accurate and did not differ significantly for target $(M=93.6 \% ; \mathrm{SD}=3.4)$ and non-target items $(M=93.8 \%$; SD $=4.0$, respectively), $F(1,16)<1$, ns.

\section{Event-related potentials}

Early differential ERP activity (150-220 ms)

Scalp potential. As shown in Figs. 1-3, an early differential processing of target compared to non-target images was found. Specifically, the calculation of Student's $t$ tests revealed a significant positive polarity shift in the ERP waveform for target compared to non-target pictures over frontal and centro-parietal sites in the time windows from 152 to $229 \mathrm{~ms}$ and 152 to $223 \mathrm{~ms}$, respectively ( $P \mathrm{~s}<0.01$; see $t$ test values in Fig. 2B). Furthermore, target images were associated with a significant negative polarity shift over temporo-occipital regions in a time window from 147 to $230 \mathrm{~ms}(P<0.01$; see $t$ test values in Fig. 2B).

Furthermore, the calculation of repeated measures ANOVA confirmed these findings. Specifically, target images were associated with a significantly enlarged negativity over temporo-occipital 

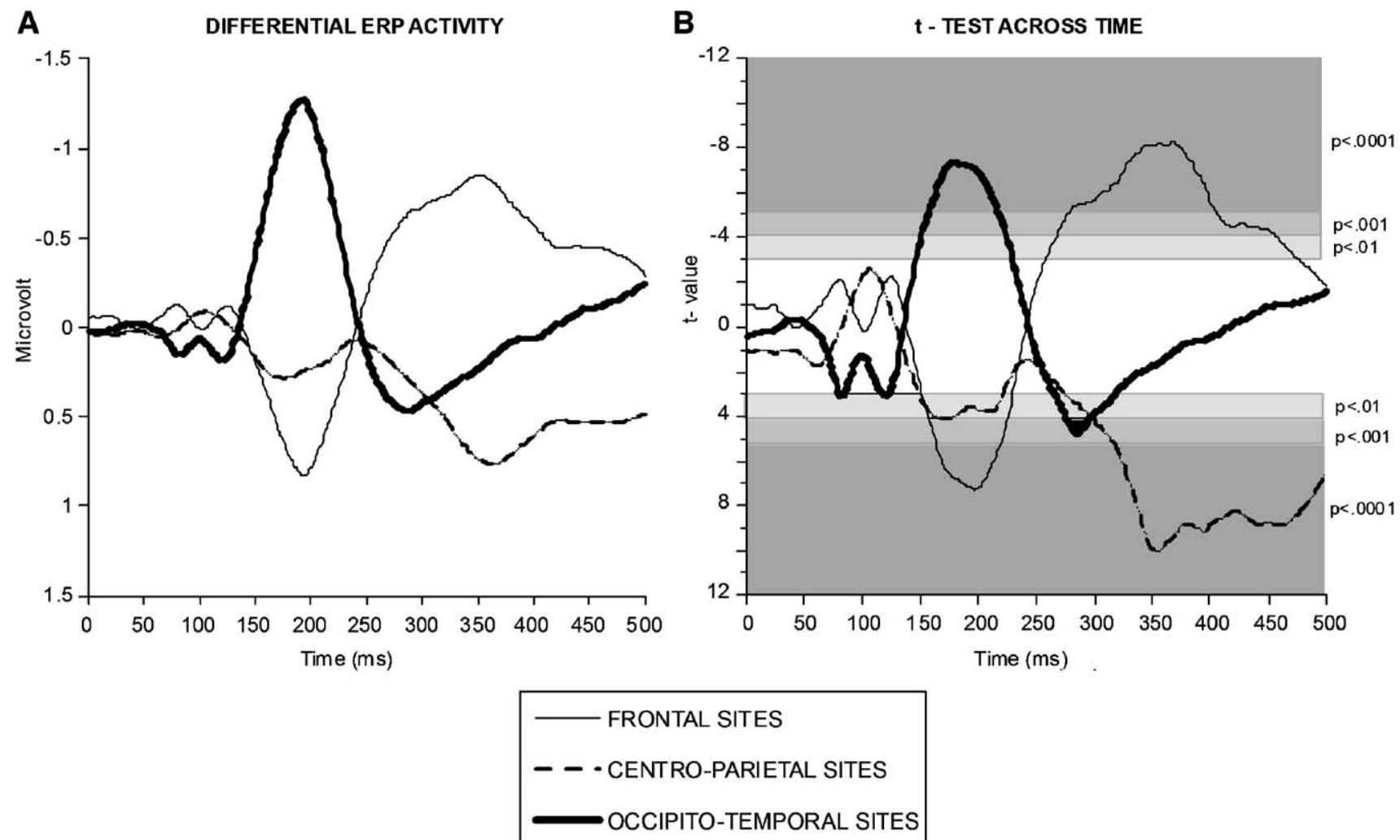

Fig. 2. (A) Illustration of the early differential ERP averaged over frontal, centro-parietal and occipito-temporal sensor sites. (B) Corresponding outcome of the statistical analysis of the difference waveforms is shown in panel A. Because there were no meaningful differences for left and right hemispheric sensor sites, waveform and statistical effects represent the average across both hemispheres.

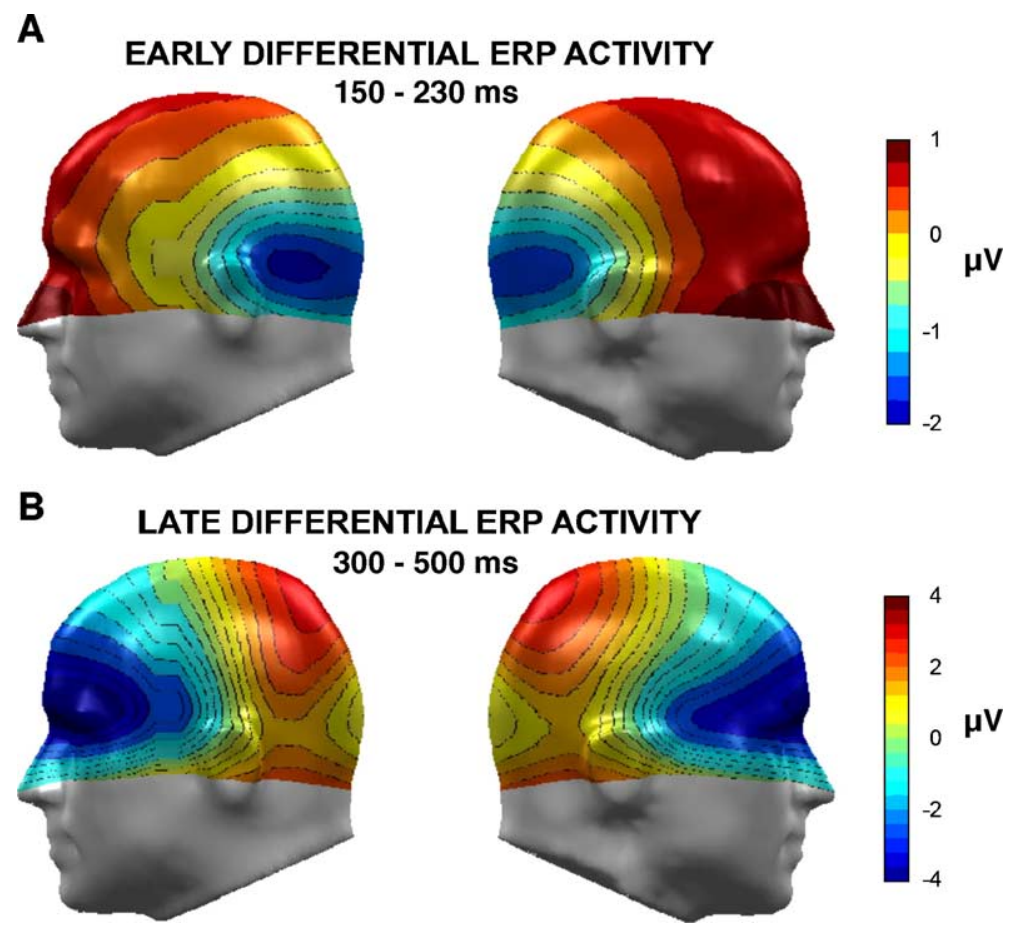

Fig. 3. (A) Scalp potential maps reveal the topography of the early differential ERP activity. Bilateral foci of temporo-occipital negativity appeared for the difference between target and non-target ERP activity averaged across the time window from 150 to 230 ms. Furthermore, polarity reversal of the early differential ERP activity is observed over centro-medial sites. In order to derive these brain maps, we interpolated voltages to the scalp surface using spherical splines and back-projected to a model head. Illustrated is a left and right side view of the model head. (B) Illustration of the late differential ERP (target-nontarget) in the classical P3 window. As expected, compared to non-target images, target pictures elicit a pronounced positive potential, maximally pronounced over centro-parietal sensor sites. 


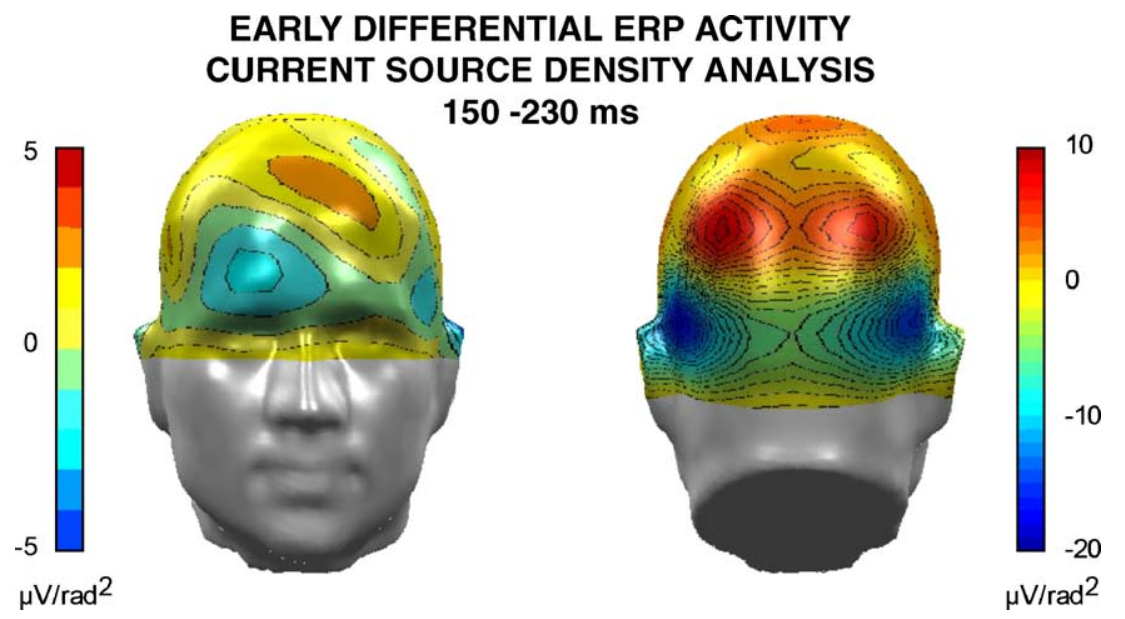

Fig. 4. Illustration of the CSD analyses for the early differential ERP activity. Illustrated are front and back views of a model head to illuminate the contributions of sink/source patterns over posterior and anterior regions. Please note that the front and back views are shown on different scales to adjust for differences in effect magnitude.

sites compared to non-target images, $F(1,16)=47.4, P<0.0001$, and stronger positivity over frontal and central sites, $F \mathrm{~s}(1,16)=$ 42.9 and 16.0, $P<0.001$, respectively; thus, resulting in a significant stimulus category by site interaction in the overall analysis, $F(2,15)=23.4, P<0.001$. In addition, no effects including the factor laterality were observed, $F \mathrm{~s}<2$.

Source estimation. Interpretation of the scalp field potentials of the early differential ERP activity is ambiguous with regard to posterior and anterior neural generator contributions. Thus, sources estimation techniques provide critical evidence whether the early differential ERP activity is solely reflecting the selective target processing in the posterior brain or both selective target processing in anterior and posterior brain regions.

As shown in Fig. 4, the CSD analysis suggested that the early differential ERP activity reflects strong neural sources of generators in posterior brain regions as indicated by pronounced bilateral posterior patterns of current sinks and sources. The multiplicity of these sinks and sources implies extended activations in posterior networks because focal brain activity is associated with less complex and less distributed sink/source patterns. In addition, the CSD analysis (right panel) also revealed extended generator structures over anterior regions with multiple sink/source patterns. However, the anterior effect appeared of much smaller magnitude than the posterior effects.

The L2-MNE analysis confirmed the finding that the early differential ERP activity reflects strong neural sources of generators in posterior brain regions. Specifically, highly significant bilateral dipole generators were suggested over occipito-temporoparietal structures. In addition, anterior activations were also revealed, which, as was the case for the $\mathrm{CSD}$, were of much smaller magnitude than the posterior effects. To confirm the significance of the anterior effect, we statistically analyzed MMN calculation for target and non-target images for each of the distributed test dipoles in the source space. To prevent spurious findings, only effects $P<0.01$ were considered meaningful. As shown in Fig. 5, multiple anterior differential activations were observed including orbito-frontal, frontal eyefield and inferior

\section{L2-MINIMUM-NORM ANALYSIS: STATISTICAL EFFECTS}

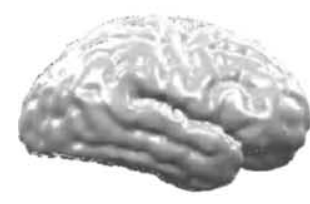

$0-150 \mathrm{~ms}$

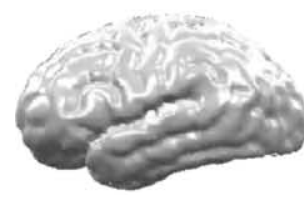

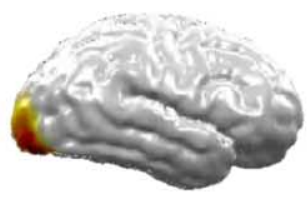

$150-175 \mathrm{~ms}$

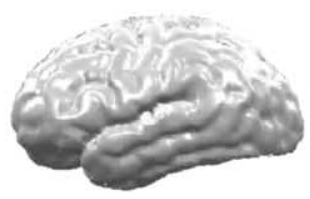

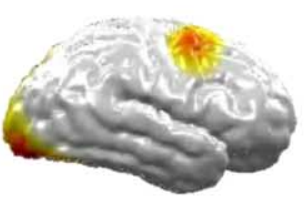

$175-200 \mathrm{~ms}$

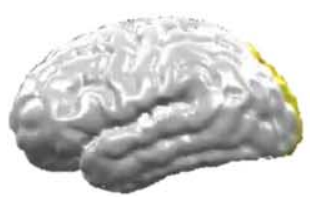

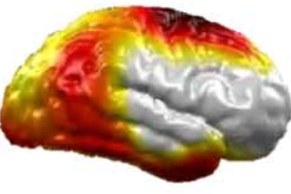

200-225 ms

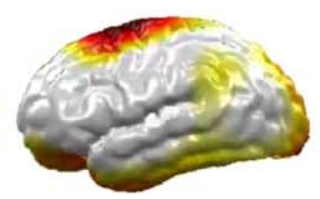

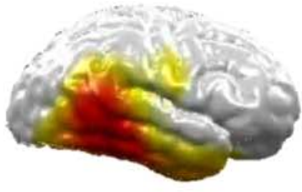

225-250 ms

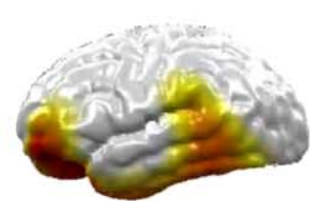

Right view

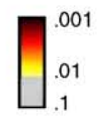

$\mathrm{p}$ - value

Left view

Fig. 5. Left and right brain model illustration of the statistical analysis of the L2-MMN analysis performed for the L2-MME solutions calculated separately for target and non-target images and each estimated generator in the source space for five time intervals. In order to avoid spurious findings, accepted level of significance was $P<0.01$. Please note that a spherical head model has been used for inverse L2-MNE modeling. The result projection on the brain model has been used in order to enhance spatial orientation. 
temporal regions. Also, a right hemisphere dominance of the neural generators was found.

\section{Late differential ERP activity: the P3 wave (300-500 ms)}

Scalp potential. Target pictures elicit a pronounced P3 wave compared to non-target pictures over centro-parietal sites, which was significant in Student's $t$ tests in a time window from 267 to $520 \mathrm{~ms}$. As shown in Figs. 2 and 3, the late differential ERP activity associated with target processing was inverted over frontal sensors, significantly modulated in a time window from 258 to 469 $\mathrm{ms}$. These findings were confirmed by conventional ANOVA analysis. A significant interaction of stimulus category and site was observed, $F(2,15)=51.6, P<0.0001$. The amplitude of the positive potential was augmented for target compared to non-target stimuli over centro-parietal sites, $F(1,16)=92.3, P<0.0001$, whereas an inverted modulation appeared over frontal sites, $F(1,16)=66.1, P<0.0001$. Furthermore, no effects including the factor laterality were observed, $F_{\mathrm{s}}<2$.

Source estimation. Both source estimation techniques suggested the contribution of multiple brain regions to the observed scalp topography of the late differential ERP activity. More specific, CSD analysis revealed pronounced bilateral sink/source patterns over lateral occipital and anterior temporal regions, which were also apparent as differential activation in the L2-MNE analysis $(P<0.01)$. However, lateralization effects, more pronounced activity over the right occipital and temporal regions, were significant in the L2-MNE analysis only $(P<0.01)$. Furthermore, the CSD analysis suggested a mid-frontal sink/source pattern, which, however, did not reach significance in the L2-MNE analysis.

\section{Discussion}

The present research examined selective attention effects in a target detection task in which target stimuli were defined by higher-order category features. Specifically, participants were asked to determine whether complex natural scenes contained an animal or not. Replicating previous findings, target and non-target ERP activity diverged sharply around $150 \mathrm{~ms}$ after stimulus onset and the early differential ERP activity appeared as positive deflection over fronto-central sensor sites and as negative deflection over temporo-occipital regions. Source analysis served to determine to what extent the early differential ERP activity reflects selective attention effects in the visual-associative cortex (i.e., inferior temporal cortex) and additional anterior generator sources possibly implicated in the expression of selective attention in posterior brain regions (cf. Bar, 2003). Both source estimation techniques (CSD and L2-MNE) suggest primary sources of the early differential ERP activity in posterior, visual-associative brain regions, and, although less pronounced, reveal the contribution of additional anterior sources. These findings concur with empirical findings from research exploring target detection tasks relying on specific stimulus features rather than higher-order features defining category membership (Rousselet et al., 2003).

Based on the findings from Delorme et al. (2004), we expected the early differential ERP activity to involve, at least in part, neural generators in the visual-associative cortex. Both source estimation techniques, CSD and L2-MNE, supported this hypothesis. Al- though neither method provides precise spatial information, both provide a reference-independent characterization of the electrical fields and allow us to infer the gross location of the neural origin of the signal. The CSD technique, which provides an estimate of local current density flowing perpendicularly to the scull into the scalp, emphasizes local cortical sources (Nunez et al., 1994).

Accordingly, as shown in Fig. 4, the pronounced bilateral sink/ source pattern over temporo-occipital sites suggests significant sources in these cortical regions. Converging evidence is provided by the minimum norm estimate, a fundamentally different source estimation method.

Using a distributed dipole model, this technique estimates an underlying source structure that would generate the measured scalp potential topography while simultaneously minimizing the overall squared generator moment. This method provides unique information regarding the location of cortical sources contributing to the measured whereas no a priori information regarding the number of generator sources is required. As shown in Fig. 5, L2-MNE analyses also reveal bilaterally pronounced differential activity in temporo-occipital regions.

The finding that the differential ERP activity elicited by target items appeared about $30 \mathrm{~ms}$ earlier over posterior compared to anterior regions provides further evidence that the fronto-central positivity does not solely reflect polarity reversal of the posterior negativity. Due to insufficient frontal electrode coverage, frontal areas are more vulnerable to spurious inverse solutions (so-called 'ghost sources') as a consequence of underestimated source covariance or overestimated signal-to-noise ratio, respectively. In order to avoid such artifacts, we applied a procedure known as regularization L-curve fitting (e.g., Hansen, 1992) minimizing ghost source appearance. The frontal generators reported here are thus unlikely to reflect spurious findings. Moreover, because of limitations inherent in each technique, we provide converging evidence across two different source estimation methods.

Recent theories specify how top-down influences may shape processing in the ventral visual pathway (Itti and Koch, 2001; Bar, 2003). It has been proposed that feature-specific enhancement of processing in the visual areas for specific categories is implemented via prefrontal connections to visual-associative cortical regions. Support for the hypothesis that the prefrontal cortex organizes top-down effects on visual processing is derived from (a) the anatomical connectivity of prefrontal regions (Selemon and Goldman-Rakic, 1988); (b) single-cell recordings showing early prefrontal activations ascending presumably from early visual areas (cf. Freedman et al., 2003); and (c) fMRI connectivity analysis providing experimental evidence for the regulation of processing in visual cortical areas by prefrontal structures (Büchel and Friston, 1997). Neuroimaging, electrophysiological, lesion and behavioral studies also suggest a dominant role of the right hemisphere in both the perceptual analysis and the recognition of faces and objects (Gauthier et al., 1999; Hasson et al., 2002; Hillger and Koenig, 1991; Kanwisher et al., 1997; Low et al., 2003; Yovel and Kanwisher, 2004; Simons et al., 2003). In accordance with these findings, our study suggested a right hemisphere dominance of the neural generators of the early differential ERP activity, which, however, was more apparent in the L2-MNE analysis. Taken together, the present findings suggest that the early differential ERP activity reflects pronounced sources in temporo-occipital cortical regions as well as modest contributions from anterior brain sites. 
A secondary aim of the present study was to explore the P3 wave associated with higher-order target categorizations. Recent studies examining the P3 wave in the context of the Go/NoGo task introduced by Thorpe et al. (1996) observed motor confounds hampering the assessment of the late differential ERP activity. Specifically, NoGo trials are associated with motor inhibition processes that affect ERP components in the time window between 320 and $500 \mathrm{~ms}$ (Macé et al., 2005; Bruin and Wijers, 2002; Rousselet et al., 2004). In order to circumvent possible confounds of the P3 wave due to motor-related processes, participants in the present study performed a two-alternative forced-choice task indicating target or non-target items with separate responses. Although reaction time distribution had a similar appearance as reported previously (e.g., Thorpe et al., 1996), it has to be noted that response times in the present study were around $80 \mathrm{~ms}$ slower compared to typical findings in previous studies with the Go/NoGo animal detection task. The longer reaction times in the present study may be attributed to the more demanding two-alternative forcedchoice task used. As expected, animal target stimuli were associated with increased P3 waves compared to non-target stimuli, in a time window beginning at $270 \mathrm{~ms}$ and lasting until $550 \mathrm{~ms}$ poststimulus. In contrast to the findings of Johnson and Olshausen (2005), the present study suggests that a P3-like potential occurs in a later time window (300 ms) and is characterized by a different scalp topography (larger positivity over centro-parietal regions for target, compared to non-target, scenes) compared to the early differential activity. Furthermore, CSD and L2-MNE analyses suggested multiple generator sites for the target P3 in bilateral occipital and anterior temporal regions, possibly also including frontal regions suggested by the CSD analysis. Thus, the findings are more consistent with the hypothesis that the early differential activity and P3 elicited by target stimuli indicate different processes invoked in the process of stimulus evaluation. Whereas the early differential ERP activity might reflect the interaction of anterior and posterior brain systems primarily involved in perceptual/sensory demands of early selection processes, the P3 is considered to reflect utilization of resources at a later and more central level of information processing (Kok, 2001). Consistent with this hypothesis, anterior temporal regions were pronounced when estimating the neural sources of the P3 but not the early differential ERP activity.

The present study examined cortical indicators of selective attention underlying categorization based on target features in natural scenes. Replicating previous findings, target compared to non-target stimuli were associated with an early differential ERP activity, which was most pronounced around 150-220 ms. Source estimation techniques suggested that the early differential ERP activity primarily reflects occipito-temporal sources. However, we also observed anterior sources implicating prefrontal structures early in the categorization process. These findings are broadly consistent with the hypothesis that feature-specific enhancement of processing in the visual areas for specific categories is implemented via prefrontal connections to visual-associative cortical regions (Bar, 2003).

\section{References}

Antal, A., Keri, S., Kovacs, G., Janka, Z., Benedek, G., 2000. Early and late components of visual categorization: an event-related potential study. Brain Res. Cogn. Brain Res. 9, 117-119.

Baas, J.M., Kenemans, J.L., Mangun, G.R., 2002. Selective attention to spatial frequency: an ERP and source localization analysis. Clin Neurophysiol. 113, 1840-1854

Bar, M., 2003. A cortical mechanism for triggering top-down facilitation in visual object recognition. J. Cogn. Neurosci. 15, 600-609.

Bruin, K.J., Wijers, A.A., 2002. Inhibition, response mode, and stimulus probability: a comparative event-related potential study. Clin. Neurophysiol. 113 (7), 1172-1182.

Büchel, C., Friston, K.J., 1997. Modulation of connectivity in visual pathways by attention: cortical interactions evaluated with structural equation modelling and fMRI. Cereb. Cortex 7, 768-778.

De Cesarei, A., Codispoti, M., Schupp, H.T., Stegagno, L., 2006. Selectively attending to natural scenes after alcohol consumption: an ERP analysis. Biol. Psychiatry 72, 35-45.

Delorme, A., Richard, G., Fabre-Thorpe, M., 2000. Ultra-rapid categorisation of natural images does not rely on colour: a study in monkeys and humans. Vision Res. 40, 2187-2200.

Delorme, A., Rousselet, G., Mace, M., Fabre-Thorpe, M., 2004. Interaction of bottom-up and top-down processing in the fast visual analysis of natural scenes. Cogn. Brain Res. 19, 103-113.

Fabre-Thorpe, M., Delorme, A., Marlot, C., Thorpe, S.J., 2001. A limit to the speed of processing in ultra-rapid visual categorisation of novel natural scenes. J. Cogn. Neurosci. 13, 171-180.

Fize, D., Boulanouar, K., Chatel, Y., Ranjeva, J.P., Fabre-Thorpe, M., Thorpe, S., 2000. Brain areas involved in rapid categorization of natural images: an event-related fMRI study. NeuroImage 11, 634-643.

Freedman, D.J., Riesenhuber, M., Poggio, T., Miller, E.K., 2003. A comparison of primate prefrontal and inferior temporal cortices during visual categorization. J. Neurosci. 23, 5235-5246.

Gauthier, I., Tarr, M.J., Anderson, A.W., Skudlarski, P., Gore, J.C., 1999. Activation of the middle fusiform 'face area' increases with expertise in recognizing novel objects. Nat. Neurosci. 2, 568-573.

Hamalainen, M.S., Ilmoniemi, R.J., 1994. Interpreting magnetic fields of the brain: minimum norm estimates. Med. Biol. Eng. Comput. 32, $35-42$.

Hansen, P.C., 1992. Analysis of discrete ill-posed problems by means of the L-curve. SIAM Rev. 34, 561-580.

Harter, M.R., Aine, C.J., 1984. Brain mechanisms of visual selective attention. In: Parasuraman, R., Davies, D.R. (Eds.), Varieties of Attention. Academic Press, New York, pp. 293-321.

Hasson, U., Levy, I., Behrmann, M., Hendler, T., Malach, R., 2002. Eccentricity bias as an organizing principle for human high-order object areas. Neuron 34, 479-490.

Hauk, O., 2004. Keep it simple: a case for using classical minimum norm estimation in the analysis of EEG and MEG data. NeuroImage 21, $1612-1621$.

Hauk, O., Keil, A., Elbert, T., Müller, M.M., 2002. Comparison of data transformation procedures to enhance topographical accuracy in time series analysis of the human EEG. J. Neurosci. Methods 113, 111-112.

Hillger, L.A., Koenig, O., 1991. Separable mechanisms in face processing: evidence from hemispheric specialization. J. Cogn. Neurosci. 3, 42-58.

Itti, L., Koch, C., 2001. Computational modeling of visual attention. Nat. Rev., Neurosci. 2 (3), 194-203.

Johnson, J.S., Olshausen, B.A., 2003. Timecourse of neural signatures of object recognition. J. Vis. 3 (7), 499-512.

Johnson, J.S., Olshausen, B.A., 2005. The earliest EEG signatures of object recognition in a cued-target task are postsensory. J. Vis. 5 (4), $299-312$.

Junghöfer, M., Elbert, T., Leiderer, P., Berg, P., Rockstroh, B., 1997. Mapping EEG potentials on the surface of the brain: a strategy for uncovering cortical sources. Brain Topogr. 9, 203-217.

Kanwisher, N., McDermott, J., Chun, M.M., 1997. The fusiform face area: a module in human extrastriate cortex specialized for face perception. J. Neurosci. 17, 4302-4311.

Kastner, S., De Weerd, P., Desimone, R., Ungerleider, L.G., 2000. Texture segregation in human extrastriate cortex: a fMRI study. J. Neurophysiol. $83,2453-2457$.

Kenemans, J.L., Kok, A., Smulders, F.T.Y., 1993. Event-related potentials 
to conjunctions of spatial frequency and orientation as a function of stimulus parameters and response requirements. Electroencephalogr. Clin. Neurophysiol. 88, 1-63.

Kok, A., 2001. On the utility of P3 amplitude as a measure of processing capacity. Psychophysiology 38, 557-577.

Low, A., Bentin, S., Rockstroh, B., Silberman, Y., Gomolla, A., Cohen, R., Elbert, T., 2003. Semantic categorization in the human brain: spatiotemporal dynamics revealed by magnetoencephalography. Psychol. Sci. 14 (4), 367-372.

Macé, M.J.M., Thorpe, S.J., Fabre-Thorpe, M., 2005. Rapid categorization of achromatic natural scenes: how robust at very low contrasts? Eur. J. Neurosci. 21, 2007-2018.

Näätänen, R., 1992. Attention and Brain Function. Lawrence Erlbaum, Hillsdale, NJ.

Nunez, P.L., Silberstein, R.B., Cadusch, P.J., Wijesinghe, R.S., Westdorp, A.F., Srinivasan, R., 1994. A theoretical and experimental study of high resolution EEG based on surface Laplacian and cortical imaging. Electroencephalogr. Clin. Neurophysiol. 90, 40-57.

Picton, T.W., Bentin, S., Berg, P., Donchin, E., Hillyard, S.A., Johnson, R., Miller, G.A., Ritter, W., Ruchkin, D.S., Rugg, M.D., Taylor, M.J., 2000. Guidelines for using human event-related potentials to study cognition: recording standards and publication criteria. Psychophysiology 37, $127-152$.

Potts, G.F., 2004. An ERP index of task relevance evaluation of visual stimuli. Brain Cogn. 56, 5-13.

Potts, G.F., Tucker, D.M., 2001. Frontal evaluation and posterior representation in target detection. Brain Res. Cogn. Brain Res. 11, 147-156.

Potts, G.F., Liotti, M., Tucker, D.M., Posner, M.I., 1996. Frontal and inferior temporal cortical activity in visual target detection: evidence from high spatially sampled event-related potentials. Brain Topogr. 9, $3-14$.

Rousselet, G.A., Fabre-Thorpe, M., Thorpe, S.J., 2002. Parallel processing in high-level categorization of natural images. Nat. Neurosci. 5, $629-630$.

Rousselet, G.A., Macé, M.J.-M., Fabre-Thorpe, M., 2003. Is it an animal?
Is it a human face? Fast processing in upright and inverted natural scenes. J. Vis. 3, 440-455.

Rousselet, G.A., Thorpe, S.J., Fabre-Thorpe, M., 2004. Processing of one, two or four natural scenes in humans: the limits of parallelism. Vision Res. 44, 877-894.

Schneider, W., Eschmann, A., Zuccolotto, A., 2002. E-Prime Reference Guide. Psychology Software Tools, Pittsburgh, PA.

Selemon, L.D., Goldman-Rakic, P.S., 1988. Common cortical and subcortical targets of the dorsolateral prefrontal and posterior parietal cortices in the rhesus monkey: evidence for a distributed neural network subserving spatially guided behavior. J. Neurosci. 8, 4049-4068.

Semlitsch, H.V., Anderer, P., Schuster, P., Presslich, O., 1986. A solution for reliable and valid reduction of ocular artifacts applied to the P300 ERP. Psychophysiology 23, 695-703.

Simons, J.S., Koutstaal, W., Prince, S., Wagner, A.D., Schacter, D.L., 2003. Neural mechanisms of visual object priming: evidence for perceptual and semantic distinctions in fusiform cortex. NeuroImage 19, 613-626.

Smid, H.G.O.M., Jakob, A., Heinze, H.J., 1999. An event-related brain potential study of visual selective attention to conjunctions of color and shape. Psychophysiology 36, 264-279.

Thorpe, S., Fize, D., Marlot, C., 1996. Speed of processing in the human visual system. Nature 6, 520-522.

Tucker, D.M., Liotti, M., Potts, G.F., Russell, G.S., Posner, M.I., 1994. Spatiotemporal analysis of brain electrical fields. Hum. Brain Mapp. 1, $134-152$.

VanRullen, R., Thorpe, S.J., 2001. Is it a bird? Is it a plane? Ultra-rapid visual categorisation of natural and artifactual objects. Perception 30, 655-668.

VanRullen, R., Thorpe, S.J., 2001. The time course of visual processing: from early perception to decision-making. J. Cogn. Neurosci. 15, $454-461$.

Wood, J.N., Grafman, J., 2003. Human prefrontal cortex: processing and representational perspectives. Nat. Rev., Neurosci. 4, 139-147.

Yovel, G., Kanwisher, N., 2004. Face perception: domain specific, not process specific. Neuron 44 (5), 889-898. 